\title{
A TRAJETÓRIA DO SISTEMA DE INFORMAÇÃO ESPECIALIZADO NA AREA DE ODONTOLOGIA (SIEO): ANTECEDENTES E AÇÕES FUTURAS ${ }^{1}$
}

\author{
Telma de Carvalho \\ Lucia Maria Sebastiana Verônica Costa Ramos \\ Vera Regina Casari Boccato \\ Cybelle de Assumpção Fontes \\ Valdete Silva Andrade \\ Cecília Isabel dos Santos \\ Izamar da Silva Freitas \\ Maria Helena Matsumoto Komasti Leves \\ Clarice Siqueira Gusso
}

\section{Resumo}

Analisou-se o Sistema de Informação Especializado na área de Odontologia (SIEO) e as mudanças ocorridas nas bibliotecas participantes a partir de sua integração ao sistema, utilizando como instrumento de coleta de dados, o questionário. Espera-se com isso identificar se as bibliotecas do SIEO foram beneficiadas pela participação na rede, se a capacitação em serviço favoreceu a formação técnica do profissional que nela atua. Pretende, ainda, verificar a contribuição do SIEO para a Rede Nacional de Informação em Ciências da Saúde, coordenada pela BIREME, bem como verificar a atuação do Centro Coordenador Nacional da área de Odontologia na administração desta rede, além de levantar considerações sobre a construção da Biblioteca Virtual em Saúde - Odontologia (BVS-O). Assim, ter-se-á maior geração de conhecimentos sobre a dinâmica do Sistema, maximizará os resultados das atividades compartilhadas, fortalecerá o SIEO e difundirá as práticas de gestão da informação para a comunidade odontológica.

Palavras chave

Sistemas de informação; Bibliotecas brasileiras de odontologia; Compartilhamento de atividades.

\section{THE TRAJECTORY OF THE SPECIALIZED INFORMATION SYSTEM IN THE AREA OF DENTISTRY: BACKGROUND AND FUTURE ACTIONS.}

\begin{abstract}
The Specialized Information System in the area of Dentistry (SIEO) and the changes in the participating libraries since they were integrated into the system were analyzed using a questionnaire as data collection instrument. With this, the expectation is to find out if the SIEO libraries benefited from the participation in the network and if the capability to provide further services has actually helped the technical education of the professional involved. It also intends to verify the SIEO contribution to the National Network of Information in Health Sciences coordinated by BIREME, as well as check the performance of the National Coordinating Center in the area of Dentistry regarding the management of this network, besides rising considerations on the construction of the Virtual Health Library of Dentistry. Thus, it will improve the generation of knowledge on the System dynamics, will maximize the results of shared activities, strengthening the SIEO, and will spread the information management practices to the dentistry community.
\end{abstract}

\section{Key-words}

Information system; Brazilian libraries of dentistry; Activity sharing.

\footnotetext{
${ }^{1}$ Trabalho apresentado no 9th International Congress Medical Librarianship - ICML9/7th Latin American and Caribbean Congress on Health Sciences Information - CRICS VII, Salvador - Brasil, 2005.
} 


\section{INTRODUÇÃO}

Os avanços tecnológicos causaram uma alteração no quadro econômico, político e social global contribuindo de forma decisiva para uma abertura e para um desenvolvimento no ramo empresarial. Nesse sentido, além de ocasionar uma crescente homogeneização cultural, a evolução e a popularização das tecnologias de informação foram fundamentais para agilizar o comércio e o fluxo de investimentos por possibilitar uma integração sem precedentes entre pontos distantes do planeta.

A evolução empresarial na era da revolução industrial se caracterizou pela Era da Produção em Massa e assim, pela Era da Eficiência. Com a Revolução da Informação, causada pelo rápido avanço da tecnologia, a Gestão da Qualidade Total, a Excelência Empresarial e a Gestão da Informação se tornaram conceitos de ordem.

O fluxo da informação ganha importância, considerando este contexto. Com as tecnologias da informação incorporadas, os fluxos da informação na empresa tendem a tornarem-se mais efetivos em virtude da elevação da capacidade de coletar, armazenar, processar e transferir informações, o que torna possível a obtenção de uma maior velocidade na comunicação, redução no prazo de respostas às variações dos ambientes, tanto interno quanto externo, melhoria na tomada de decisão, enfim, permite um aumento da eficiência organizacional em vários aspectos.

Neste sentido este trabalho pretende demonstrar a trajetória de um sistema de informação especializado na área de odontologia que atua há 11 anos prestando serviços compartilhados em benefício da comunidade odontológica brasileira.

\section{SISTEMAS DE INFORMAÇÃO NOS SERVIÇOS EM REDE}

A informação hoje é considerada como a válvula propulsora das grandes sociedades econômicas do planeta; o insumo básico para esse desenvolvimento. 
Segundo Dias e Belluzzo (2003, p. 24-25),

a informação é considerada, cada vez mais, um recurso estratégico e de valor agregado para a percepção e absorção de novas tecnologias [...] Como insumo decisório, a informação tem papel preponderante na cadeia de valores de uma organização, constituindo-se em instrumento para diagnosticar a vantagem competitiva e encontrar maneiras para intensificá-la.

A tecnologia da informação vem contribuindo para a mudança dos cenários organizacionais, mais notadamente no que se refere ao aumento da capacidade de processamento, da estrutura e dos fluxos de informação.

Dentro desse contexto, os Sistemas de Informação têm um papel de suma importância quanto ao acesso e a busca da informação.

Segundo Lima (1998, p. 8),

Sistema de Informação pode ser uma biblioteca, pública ou especializada; um centro de documentação de uma empresa; um arquivo, um museu ou um banco de dados. Seja qual for a sua denominação original, um Sistema de Informação tem por função coletar, tratar e disseminar a informação produzida pela sociedade na qual está inserido, garantindo assim, o acesso à cultura por parte de seus membros e possibilitando a sua continuidade.

Para Guimarães é o conjunto de dados e informações organizado de forma integrada, com o objetivo de atender a demanda e antecipar as necessidades dos usuários.

O Sistema de Informação apresenta duas vertentes:

- a coleta, estruturação e organização do capital informação (recursos: documentos de todo tipo);

- a colocação dessas bases ao alcance de todos, promovendo intercâmbio que agreguem valor ao capital informação/conhecimento, para fundamentar a tomada de decisões/estratégias.

Os conhecimentos da entidade vão, dessa maneira, sempre evoluindo, abrindo novas perspectivas estratégicas. A informação como elemento integrante desse Sistema de Informação tem o seu valor atribuído às condições em que esta é utilizada. 
Nesse sentido, podemos estabelecer também alguns princípios para o planejamento de um Sistema de Informação como a filosofia de informações para a organização (recursos empregados e autonomia desejada); uso da tecnologia da informação como fator estratégico, econômico e organizacional; a evolução da empresa (atualização e capacitação dos recursos).

Quanto às vantagens e as desvantagens de um Sistema de Informação, essas podem ser verificadas e analisadas tendo como parâmetros (GUIMARÃES):

\section{Vantagens:}

- registro legível dos dados;

- aumento da produtividade;

- redução de erros no registro dos dados;

- registro do horário correto de entrada dos dados;

- produção de relatórios específicos;

- redução de documentos;

- produção de conhecimento através da utilização das informações em atividades de ensino e pesquisa;

- uso sistemático do sistema.

Desvantagens:

- falhas na condução do sistema;

- quebra da privacidade e segurança dos dados;

- número inadequado de equipamentos;

- necessidade de constante atualização (máquina, informação, usuário).

A conscientização do usuário, na visão de Borges (1995, p. 187-188), juntamente com a "[...] compreensão de que a informação é um fator que deve ser conhecido, processado, compreendido e utilizado pela consolidação de serviços, produtos e sistemas de 
informação [...]", contribui para ampliar, de forma segura, as atividades humanas quer na área sociocultural, quer na econômica.

A área de odontologia possui uma rede denominada Sistema de Informação Especializado na área de Odontologia (SIEO) que atua com as características de um Sistema de Informação acima. Este trabalho que pretende tecer considerações sobre a trajetória deste Sistema de Informação que já atua há 11 (onze) anos na área de odontologia e que é constituído por Instituições de Ensino Superior Estaduais e Federais, ligadas à Rede Brasileira de Informação em Ciências da Saúde, do Centro Latino-Americano e do Caribe de Informação em Ciências da Saúde - BIREME.

\section{METODOLOGIA}

Para a realização deste estudo exploratório foi utilizado como instrumento de coleta de dados o questionário, formado por seis questões abertas, que permitiu aos bibliotecários responsáveis pelas atividades realizadas no Sistema de Informação Especializado na área de Odontologia manifestarem-se a respeito da sua visão sobre crescimento da instituição e do seu próprio desenvolvimento profissional a partir da formação desta rede, bem como tecer considerações sobre o trabalho de administração desenvolvido pelo Centro Coordenador e das expectativas em relação à criação da Biblioteca Virtual em Odontologia.

\subsection{Caracterização institucional: Sub-Rede Nacional de Informação na Área de Ciências da Saúde Oral}

Em 1991, através de Termo Aditivo ao Convênio BIREME/USP firmado em 1990, o SDO tornou-se o "Centro Coordenador Nacional de Informação em Ciências da Saúde Oral”.

Deste convênio originou-se o projeto de "Implementação de rede de informação na área odontológica", apresentado à W. K. Kellogg Foundation em 1990 e aprovado em 1991 em parceria com a BIREME (Centro Latino Americano e do Caribe em Ciências da Saúde), 
sendo a Universidade de São Paulo representada pela Faculdade de Odontologia da USP e o seu Serviço de Documentação Odontológica - SDO².

Tendo o SDO a responsabilidade de localizar, no território nacional, bibliotecas de Odontologia que dispusessem de acervo bibliográfico adequado, recursos humanos que pudessem atender à demanda dos serviços que seriam oferecidos à comunidade e cursos de pós-graduação strictu senso ou doutorado, inicialmente a Sub-Rede Nacional de Informação na Área de Ciências da Saúde Oral, contou com 6 Universidades brasileiras, constituindo-se os "núcleos básicos de odontologia".

Para se chegar aos seis primeiros núcleos participantes da Sub-Rede, conforme Carvalho (1999), foram diagnosticadas as Universidades Estaduais e Federais Brasileiras, através da utilização do serviço de comutação bibliográfica e de questionários encaminhados às instituições. A localização regional também foi outro fator que contribuiu para a definição dos núcleos que a integrariam. A partir das análises documentais efetuadas, deu-se início a contatos com as instituições, por meio de visitas direcionadas aos locais, analisando-se "in loco" as potencialidades das Instituições de Ensino Superior representadas por suas bibliotecas.

Posteriormente, foram elaborados "Relatórios de Viabilidade de Participação", dessas universidades, ao projeto em questão e assinados os "Termos de Ajuste de Cooperação Técnica" entre os participantes da Sub-Rede.

Ainda, conforme Carvalho (1999) em 1992, novo projeto foi encaminhado a W. K. Kellogg Foundation, onde se previa a criação de mais 14 núcleos de odontologia, subdivididos em 4 básicos e 10 de apoio.

\footnotetext{
${ }^{2}$ KRZYZANOWSKI, Rosaly Favero. Implementação de rede de informação na área odontológica. São Paulo, 1990. (Projeto apresentado à W. K. Kellogg Foundation em parceria com a BIREME - Centro Latino-Americano e do Caribe em Ciências da Saúde).
} 


\section{COMUNICACÃO}

Por "núcleo básico" entendia-se a unidade de informação que tinha como responsabilidades:

1) efetuar a busca bibliográfica por meio de CD-ROM;

2) agilizar o serviço de comutação on line utilizando-se do software desenvolvido pela BIREME;

3) reunir a literatura odontológica regional para indexação nas bases de dados LILACS/BBO;

4) divulgar os serviços oferecidos pela Sub-Rede.

Por "núcleo de apoio" entendia-se a unidade de informação que tinha como responsabilidades:

1) efetuar a busca bibliográfica nas bases de dados LILACS/BBO;

2) efetuar a solicitação de busca bibliográfica pelo serviço de comutação online utilizando-se do software desenvolvido pela BIREME, como solicitantes;

3) divulgar os serviços oferecidos pela Sub-Rede.

Para que o SDO pudesse levar a efeito as novas atividades advindas do convênio - tais como: indexação da literatura odontológica nacional através de metodologia própria desenvolvida pela BIREME, em linguagem Microisis (Metodologia LILACS), utilização do serviço de comutação bibliográfica on-line, realização de levantamento bibliográfico utilizando base de dados em CD-ROM, dotou seu acervo de recursos bibliográficos, capacitou sua equipe bibliotecária por meio de cursos/treinamentos referentes aos aplicativos que seriam utilizados para realização das tarefas bem como de conhecimentos específicos de informática e do software utilizado pela rede local instalada na biblioteca.

A partir do aprimoramento dos recursos humanos do SDO pelo treinamento oferecido pela BIREME na utilização de seus aplicativos, o SDO tornou-se o "multiplicador" das informações aos núcleos básicos de odontologia. 
Carvalho (1999) salienta ainda que as bibliotecas dos seis Estados brasileiros que formaram os primeiros núcleos tiveram treinamento formal nas dependências do SDO, o mesmo acontecendo posteriormente com os outros quatro núcleos básicos.

Em termos de equipamentos de informática, o projeto encaminhado à W. K. Kellogg Foundation possibilitou ao SDO a formação de sua rede local bem como dotou os integrantes do sistema de equipamentos que permitissem operacionalizar as atividades propostas no convênio, além de conceder pelo Centro Coordenador, a assinatura das bases de dados LILACS/BBO e MEDLINE aos integrantes do sistema, por um ano.

Inicialmente, a Sub-Rede Nacional de Informação na Área de Ciências da Saúde Oral contou com a participação de 21 Instituições de Ensino Superior Estaduais e Federais e tinham por objetivo principal levar aos pesquisadores o que havia de mais recente na literatura odontológica, oferecendo condições de localização e recuperação dessas informações de uma forma mais ágil e segura.

O Quadro 1 demonstra a distribuição da Sub-Rede Nacional de Informação na área de Ciências da Saúde Oral:

QUADRO 1 - DISTRIBUIÇÃo DA SUB-REDE NACIONAL DE INFORMAÇÃO NA ÁREA DE CIÊNCIAS DA SAÚDE

\begin{tabular}{|c|c|c|}
\hline IESE & IESF & OUTROS \\
\hline UNESP- Araçatuba* & UFRS* & Rede Cedros ${ }^{\bullet}$ \\
\hline USP - Bauru* & UFRJ* & \\
\hline USP - São Paulo (SDO) ${ }^{\bullet}$ & UFBA* & \\
\hline USP - Ribeirão Preto ${ }^{\star}$ & UFPR* & \\
\hline Universidade de Pernambuco.* & UFMG* & \\
\hline UNICAMP - Piracicaba ${ }^{\bullet}$ & UFMS* & \\
\hline \multirow{7}{*}{ Universidade Estadual de Pelotas/RS ${ }^{*}$} & UFRN* & \\
\hline & $\mathrm{UFF}^{*}$ & \\
\hline & UF Ponta Grossa ${ }^{\bullet}$ & \\
\hline & $\mathrm{UFMA}^{\bullet}$ & \\
\hline & $\mathrm{UFPB}^{*}$ & \\
\hline & $\mathrm{UFPA}^{*}$ & \\
\hline & $\begin{array}{l}\text { Faculdade Federal de Odontologia de } \\
\text { Diamantina (FAFEOD) }\end{array}$ & \\
\hline $\mathrm{N}=7$ & $\mathrm{~N}=13$ & $\mathrm{~N}=1$ \\
\hline
\end{tabular}

$\mathrm{N}=7$

* Núcleo Básico

- Núcleo de apoio 


\subsection{O Sistema de Informação Especializado na área de Odontologia (SIEO)}

Houve mudança na designação do nome da Sub-Rede Nacional de Informação na área da Saúde Oral uma vez que deixaram de existir as figuras dos núcleos de apoio e dos núcleos básicos já que, passados alguns anos, a maioria deles exerciam atividades como centros cooperantes.

Algumas das funções exercidas anteriormente pelos núcleos de apoio deixaram de responder às necessidades da época, mas hoje algumas destas instituições poderiam contribuir com a construção da Biblioteca Virtual em Odontologia, fortalecendo o projeto e retomando sua participação nesta rede. Atualmente o Sistema de Informação Especializado na área de Odontologia é composto pelas seguintes instituições de ensino superior estaduais e federais:

QUADRO 2 - DISTRIBUIÇÃO DO SISTEMA DE INFORMAÇÃO ESPECIALIZADO NA ÁREA DE ODONTOLOGIA

\begin{tabular}{ll}
\hline \multicolumn{1}{c}{ IESE } & IESF \\
\hline UNESP- Araçatuba, Araraquara e São José & UFRGS* \\
dos Campos* & UFRJ* \\
USP - Bauru* & UFBA* $^{*}$ \\
USP - São Paulo (SDO) & UFPR* $^{*}$ \\
UPE - Universidade de Pernambuco- & UFMG* $^{*}$ \\
Faculdade de Odontologia* & UFMS* $^{*}$ \\
& UFRN* $^{*}$ \\
& \\
\hline $\mathrm{N}=6$ & $\mathrm{~N}=7 \%$ \\
\hline
\end{tabular}

- Centro Coordenador

* Centros Cooperantes 
O questionário foi encaminhado a 12 (doze) instituições de ensino superior que compõem o SIEO por e-mail próprio da rede de odontologia e obteve-se o retorno de 8 (oito) questionários correspondendo a $66,67 \%$ de questionários respondidos. O Serviço de Documentação Odontológica, como Centro Coordenador, não foi objeto de pesquisa.

\section{RESULTADOS}

Com a análise das respostas recebidas pelos responsáveis pelas atividades do SIEO pode-se tecer os seguintes comentários:

\subsection{Quanto à Formação da Rede de Odontologia}

Pelas respostas analisadas a formação do SIEO foi uma boa iniciativa por permitir a descentralização de funções e proporcionar um trabalho cooperativo e compartilhado representado pela concretização das metas e dos objetivos da rede. A integração dos bibliotecários da área foi outro ponto destacado em relação à formação do SIEO com destaque para o bom relacionamento entre os profissionais. As atividades de localização e inclusão de documentos científicos em diversas regiões do país nas bases de dados BBO e LILACS favoreceram o desenvolvimento de produtos com qualidade para quem utiliza os serviços prestados pela rede. A formação do SIEO foi considerada, ainda, "muito produtiva e com grandes oportunidades de evoluir cada vez mais".

\subsection{Quanto ao Favorecimento dos Clientes a Partir da Participação da Instituição na Rede de Odontologia}

Vários itens foram assinalados neste ponto, entre eles a vantagem da biblioteca possuir um bibliotecário específico para a área de odontologia uma vez que algumas atuam em área biomédica e/ou saúde. Além disso, a rede favoreceu os clientes de forma positiva e proporcionou também maior visibilidade dos produtos e serviços o que gerou um “crescimento vertiginoso da demanda" pela melhor orientação aos usuários. 
A disponibilização de maior quantidade de informações nas bases de dados e o atendimento da comutação bibliográfica também foram considerados como fatores relevantes para o favorecimento dos clientes. A rede proporcionou maior visibilidade dos trabalhos defendidos em cada instituição pela sua inclusão nos bancos de dados.

Outro ponto que mereceu destaque foi a informação recebida por uma das bibliotecas em relação aos clientes de que houve favorecimento financeiro na solicitação de artigos para fora da instituição e melhoria da busca bibliográfica em função do aumento do conhecimento do bibliotecário sobre indexação. Como destaque, ainda, pode-se considerar também o oferecimento de treinamentos de serviços e produtos.

\subsection{Quanto ao Desenvolvimento e Aprimoramento Profissional e Técnico do Bibliotecário}

Todos foram unânimes ao responderem que a rede favoreceu os seus aprimoramentos profissionais, ligados ao processo e tratamento da informação, ao conhecimento de como utilizar as ferramentas de trabalho na alimentação da BVS, a manipular e disseminar novas tecnologias de informação. Foi salientado, também, que a capacitação contínua propicia a melhoria no atendimento às necessidades informacionais dos clientes e que este trabalho em rede exige atualização constante do profisssional por trabalhar com tecnologia altamente especializada.

\subsection{Quanto à Contribuição da Biblioteca no SIEO}

A maioria dos respondentes revelou que possuem um pequeno quadro de pessoal e este fator é o que mais provoca o que consideraram como "participação pequena, porém de forma eficaz e direta para o crescimento do sistema”. A grande contribuição da rede é na alimentação das bases de dados e na comutação bibliográfica. Foi salientado que "os integrantes assumem diferentes níveis de participação em atividades cooperativas utilizando toda a potencialidade informacional da rede". 


\subsection{Quanto às Sugestões para o Centro Coordenador}

A capacitação profissional dos bibliotecários foi o item mais solicitado para que o Centro Coordenador possa prover. Diversas sugestões foram dadas para este fim como: desenvolvimento de palestras e seminários por região, ou ainda, criação de listas de discussões com temas específicos e com especialistas do Centro Coordenador, seminários, encontros, estágios profissionais. O uso de tecnologias como videoconferência, áudioconferência foi evidenciado como possibilidade de atendimento a estas questões de capacitação.

Outras sugestões também foram recebidas como a de estimular o atendimento prioritário dos usuários do SIEO com criação de políticas específicas para este fim. Manter a integração bem como as parcerias também obtiveram destaque, além de solicitação para resgatar a elaboração de material de divulgação da rede. A maioria respondeu que o SIEO é bem administrado, atendendo prontamente as solicitações de esclarecimentos de dúvidas.

Uma das respostas aponta que deveria haver maior contato com o grupo principalmente nesta fase de construção da BVS Odontologia. A simpatia e cordialidade da equipe do Centro Coordenador também foi mencionada como motivador para o trabalho em rede.

\subsection{Quanto à Construção da Biblioteca Virtual em Saúde em Odontologia}

Nesta questão a maioria dos respondentes se ateve aos benefícios que a construção da BVS Odontologia está proporcionando, como: "um empreendimento de grande importância na área, pois dará visibilidade à mesma", ou ainda "fonte de informação inesgotável na área da saúde oral", ou "proporcionar um grande benefício a todos os usuários da comunidade científica odontológica nacional e internacional quanto ao acesso e recuperação da informação de responsabilidade e disponibilizadas pelo SIEO”.

Também são unânimes quanto aos benefícios que este projeto trará para o usuário, para o profissional da informação, para a unidade de informação, para o SIEO e para a BVS como 
um todo. Apenas um bibliotecário respondeu que "falta treinamento" para trabalhar com a BVS.

Houve menção, também, sobre a união dos integrantes do Comitê Consultivo da BVS Odontologia, do Centro Coordenador e dos Centros Cooperantes para o andamento deste projeto, todos muito engajados em fazer o melhor, mesmo com as limitações de cada centro.

\section{CONCLUSÕES}

O trabalho desenvolvido pelos profissionais que atuam no SIEO atesta a seriedade desta rede de odontologia que vem se consolidando ao longo de seus onze anos de funcionamento.

O espírito de equipe e a interação das pessoas é um motivador para o bom andamento das atividades.

Apesar da falta de pessoal o SIEO consolida-se como um sistema de informação atuante e seus Centros Cooperantes têm expressiva contribuição na Rede Brasileira de Informação em Ciências da Saúde Oral.

A Biblioteca Virtual em Odontologia, em fase de desenvolvimento, conta com o empenho de todos os seus componentes para que o projeto se concretize o mais rápido possível em beneficio da comunidade odontológica. Este projeto aproximou e envolveu a todos que lutam e anseiam por ele: bibliotecários do SIEO, Centro Coordenador, Comitê Consultivo e a própria BIREME. 


\section{REFERÊNCIAS}

BORGES, M. E. N. A informação como recurso gerencial das organizações na sociedade do conhecimento. Ciência da Informação, Brasília, v. 24, n. 2, p. 181-188, maio/ago. 1995.

CARVALHO, T. Parâmetros para análise ambiental da sub-rede nacional de informação em ciências da saúde oral. 1999. 120 f. Dissertação (Mestrado) - Pontifícia Universidade Católica de Campinas, Campinas, 1999.

DIAS, M. M. K; BELLUZZO, R. C. B. Gestão da informação em ciência e tecnologia sob a ótica do cliente. Bauru: EDUSC, 2003. 184 p.

GUIMARÃES, E. M. P. Sistema de informação em saúde. Disponível em: $<$ www.enf.ufmg.br/disciplinas/sisinfosaude.ppt $>$. Acesso em: 24 mar. 2005.

LIMA, V. M. A. Terminologia, comunicação e representação documentária.1998.116 f. Dissertação (Mestrado em Ciências da Comunicação) - Escola de Comunicações e Artes, Universidade de São Paulo, São Paulo, 1998. 


\section{COMUNICAČ̃̃O}

\section{Telma de Carvalho}

Diretora Técnica do Serviço de Documentação Odontológica da Faculdade de Odontologia da Universidade de São Paulo - SDO/FO-USP e doutoranda do Programa de Pós-graduação em Ciências da Comunicação da Escola de Comunicações e Artes da Universidade de São Paulo ECA-USP

telmac@usp.br

\section{Lucia Maria Sebastiana Verônica Costa Ramos}

Supervisora Técnica do Serviço de Documentação Odontológica da Faculdade de Odontologia da Universidade de São Paulo - SDO/FO-USP

ferpau@usp.br

\section{Vera Regina Casari Boccato}

Bibliotecária do Serviço de Biblioteca e Documentação da Faculdade de Odontologia de Bauru da Universidade de São Paulo - SBD/FOB-USP e mestranda do Programa de Pós-graduação em Ciência da Informação da Universidade Estadual Paulista- UNESP, Campus de Marília vboccato@fob.usp.br

\section{Cybelle de Assumpção Fontes}

Diretora Técnica do Serviço de Biblioteca e Documentação da Faculdade de Odontologia de Bauru da Universidade de São Paulo - SBD/FOB-USP e Mestre em Ciências da Comunicação da Escola de Comunicações e Artes da Universidade de São Paulo - ECA-USP

cafy@fob.usp.br

\section{Valdete da Silva Andrade}

UFBA - valdete@ufba.br

\section{Cecilia Isabel dos Santos}

Bibliotecária da Universidade Federal do Rio Grande do Norte - UFRN

cecilia@dod.ufrn.br

\section{Izamar da Silva Freitas}

Diretora da Biblioteca da Faculdade de Odontologia de Araçatuba da Universidade Estadual Paulista - FOA-UNESP

izamar@foa.unesp.br

\section{Maria Helena Matsumoto K. Leves}

Diretora da Biblioteca da Faculdade de Odontologia de Araraquara da Universidade Estadual

Paulista - FOAr-UNESP

dirstbd@foa.unesp.br

\section{Clarice Siqueira Gusso}

Diretora da Biblioteca de Ciências da Saúde - Sede Botânico da Universidade Federal do Paraná UFPR

bibsb@ufpr.br

Artigo aceito para publicação em janeiro de 2006. 